\title{
Combined friction and wear reduction in a reciprocating contact through laser surface texturing
}

\author{
Sorin-Cristian Vlădescu, ${ }^{a, *}$, Andrew V. Olver ${ }^{\mathrm{a}}$, Ian G.Pegg ${ }^{\mathrm{b}}$, Tom Reddyhoff ${ }^{\mathrm{a}}$ \\ ${ }^{a}$ Tribology Group, Department of Mechanical Engineering, Imperial College London, South Kensington, Exhibition \\ Road, SW7 2AZ, London, United Kingdom \\ ${ }^{\mathrm{b}}$ Ford Motor Company, Dunton, Essex, United Kingdom \\ * Corresponding author. Tel.: +44 (0) 2075947236 \\ E-mail address: s.vladescu12@imperial.ac.uk
}

\begin{abstract}
The aim of this study is to gain insights into the interactions between laser-textured surface pockets and friction and wear behaviour of an automotive piston-liner pairing. To do this, a recently developed, reciprocating, test apparatus was used to conduct wear tests under highly loaded conditions. Fused silica specimens with a range of pocket geometries were rubbed against a convex steel pad and the resulting friction and wear data were compared with those from a non-textured specimen. Contact conditions were set to remove the influence of initial surface roughness on texture behaviour. These tests showed that, as the specimen become worn and surface roughness increases, the contact progresses further into the mixed and boundary regime. This leads to a significant improvement in the relative performance of the textured specimens, showing reductions in friction of up to $70 \%$, compared with the non-texture case. This is consistent with previous results that have shown texture to have the effect of boosting film thickness in the mixed lubrication regime. Surface texture was also shown to reduce the volume of wear, by up to 69\% (corresponding to a change in wear coefficient from $2.67 e^{-4}$ to $0.81 e^{-4}\left[\mathrm{~mm}^{3} / \mathrm{N} \cdot \mathrm{m}\right]$ ). Another, important, finding is that both friction and wear reduce monotonically as the sum of the pocket volumes along the stroke increases. This may aid texture design, since it means that individual pocket width and depth values can largely be ignored, so long as the overall volume is maximised. The only exception to this trend is when the pockets are larger than the contact area. In this case, friction increases due to a collapse of the lubricant film, while wear reduction remains unaffected - a discrepancy which may suggest that pockets reduce wear and friction via separate mechanisms
\end{abstract}

\section{Introduction}

Surface texture has been investigated as a means of controlling friction and wear in sliding contacts for approximately fifty years [1], since it is relatively simple to implement and may be used in conjunction with other lubrication approaches. Currently, surface texture is receiving increased attention due to the need to improve the energy efficiency of automotive contacts [2], which, in turn, is driven by stricter $\mathrm{CO}_{2}$ emission limits [3], and the pressing issues of climate change. It is also the case that methods of producing texture are becoming cheaper and more accurate [4]. Despite all this, there is still a lack of firm evidence regarding the mechanisms by which surface texture affects tribological performance and this is limiting its development and application. 
Investigations into how surface texture functions can typically be divided between those that relate to friction and those that relate to wear. The majority of early texture friction experiments were performed under full film hydrodynamic and elastohydrodynamic conditions [5-15]. Under these regimes, no conclusive improvement due to surface texture was demonstrated (with different studies showed the effectiveness of texture as being beneficial [13][Morris], detrimental [14], negligible [15]). Recently, however, more attention has been paid to measuring texture performance under mixed and boundary conditions, where several studies [16-21], have shown how pockets can lead to friction reductions of more than 50\%. Furthermore, such conditions of mixed and boundary lubrication have become increasingly prevalent due to the recent trend towards lower viscosity lubricants aimed at reducing hydrodynamic losses [22].

The main explanations of how surface texture affects friction can be summarised as follows. In 1966 Hamilton and Allen [1,23], introduced the concept of "micro-irregularities" and suggested that surface texture can create resistance that acts to prevent fluid from escaping the contact. Following this, Tønder proposed that surface patterns generate a "virtual step", within the contact that resembles the discontinuous change in film thickness associated with a Rayleigh step bearing [24,25]. Later, when studying the behaviour of transverse roughness in EHL lubricated contacts, Morales-Espejel [26] and Greenwood [27] suggested that each asperity peak entering contact zone behaves as a flow exciter that closes and opens the inlet to allow varying amounts of fluid to enter. Years later, Morales-Espejel, Tripp and co-workers [28] observed that, under EHL lubrication conditions, oil is released from the pocket under pressure, as a result of elastic deformation of the material in the contact area, and this could increase load support and hence film thickness. In 2006, the concept of "inlet suction" was identified in Olver and Fowell's work [29,30], whereby the reduction in pressure afforded by cavitation as lubricant expands into a pocket at the contact inlet leads to an increase in lubricant entrainment and hence film thickness. Very few of these mechanisms have been proved experimentally. In terms of experimental evidence, what is known so far is that pockets boost film thickness in the mixed and boundary regime [31], typically by a few tens of nanometres, which, due to the slope of the Stribeck curve in this lubrication regime, has a significant effect on friction [32]. Furthermore, a single pocket entrainment causes only a temporary increase in film thickness, which must be sustained by the entrainment of subsequent pockets otherwise it will become squeezed from the contact.

Studies into the effects of surface texture on wear have uncovered two main mechanisms, namely those of "lubricant replenishment" and "wear debris trapping". The first of these relates to starvation i.e. the reduction in film thickness due to an inadequate volume of lubricant present at the inlet [33], which is a believed to be an issue in automotive piston-liner contacts, caused by the rapid reciprocating motion of the contact and the fact that the oil must lubricate multiple rig contacts [34]. Starvation occurs when lubricant is pushed aside during one pass of the contact and does not flow back in time for the next pass [33]. In such situations, pockets on component surface can act as "micro-reservoirs" and increase the flow of lubricant back to the contact - a process known as 'lubricant replenishment' [35]. Several studies have investigated this mechanism. Blatter et al. [36] ran pin-on-disc tests on specimens with varying groove geometries, showing reductions in friction coefficient and wear rate, coupled with the increased wear life that were attributed to the grooves' ability to store lubricant and replenish the track. In a more recent study, Borghi et al. [37] examined the chemical composition of textured and non-textured surfaces using Energy Dispersive Spectroscopy (EDS). This revealed significant quantities of $\mathrm{Mg}$ and $\mathrm{Ca}$ inside the dimples, thus demonstrating the retention of lubricant as the means by which the textured surfaces reduced friction and wear. Krupka et al. [38] also concluded that pockets can act as oil reservoirs capable of supplying lubricant into the contact region to overcome starvation. Their tests, carried out under EHL and mixed lubrication conditions, showed micro-dimpled surfaces overcame starvation and increased film thickness, which resulted in a measurable reduction in abrasive 
wear. Further important work suggesting that pockets reduce friction by acting as reservoirs for lubricants, and hence aiding a "micro-wedge" effect, has been provided by Rahnejat and co-workers. Finally, the current authors observed that pockets carried lubricant into the cavitated region, and suggested that this could reduce starvation in reciprocating contacts, in which the cavitated region would otherwise be ingested by the inlet following reversal [16].

The second mechanism proposed to explain texture induced wear reduction is that of "debris trapping" whereby pockets remove particles, which would otherwise remain in the contact and accelerate surface damage. One of the first studies into this was carried out by Varenberg et al. using a flat-on-flat sliding contact under dry fretting conditions [39]. They showed wear debris being removed from the contact area and accumulating in the micro-textured pockets. Following this, they showed schematically how the pocket filling takes place: starting at the rim, followed by the centre and finally the base. In addition to this, Pettersson and Jacobson investigated the influence of surface texture on wear under starved and lubricated boundary conditions $[18,19]$ and concluded that recesses can remove debris from the contact zone and hence delay or decelerate wear [19]. They used a reciprocating ballon-plate tribometer and observed that grooves and squares parallel to the sliding direction reduced wear to negligible levels compared to an untextured surface. Further confirmation of pockets acting as debris traps has been obtained through the use of relatively recent techniques, such as SEM, that allow the morphology and chemistry of textured surfaces to be captured immediately after performing tribological tests. The first of these studies was carried out by Zum Gahr et. al [40] on plain and micro-textured steel/oxide-ceramic and self-mated oxide-ceramics sliding pairs. They noticed that instantaneous spikes in the friction force signal were present after $50 \mathrm{~m}$ of sliding for the untextured specimens but did not appear until after $400 \mathrm{~m}$ of sliding for the dimpled textured specimens. SEM micrographs of the worn surfaces obtained after the test showed that both fresh and partially compacted debris from the contact area had accumulated in the micro-dimples. Their conclusion was that the friction spikes observed early on in the untextured specimens sliding tests were due particle entrapment, whereas the eventual spikes in the texture surface tests were due to the detachment of layers, or groups, of wear particles which had accumulated in the micro-dimples.

As described above, the majority of investigations into surface texture have, so far, focussed on either friction or wear but not both simultaneously. Since texture is most effective in reducing friction under mixed and boundary conditions, where appreciable surface contact and wear occurs, it seems likely that the effects of pockets on friction and wear are interlinked. The aim of the simple experiments described in this study is to explore the relationship between these three parameters. We hope that this will provide fundamental insights which will inform how deliberately applied surface texture can improve the lubrication of piston/cylinder contacts, since these account for $45 \%$ of the frictional energy dissipated in a typical automobile (frictional losses in turn make up $11.5 \%$ of the total fuel energy [2]). Furthermore, for the reasons mentioned above, it is believed that contacts in these components experience starvation and may therefore benefit from texture-enhanced lubricant replenishment. That being said, our findings may also be applied to other vehicle components, such as crankshaft bearings (which consume around 19\% of a vehicle's fuel energy [41]), as well as other conformal contacts in application such as compressors. 


\section{Experimental procedure}

\subsection{Test rig description}

This study used a custom-built reciprocating test rig capable of measuring friction force under conditions that elosely partially replicate those found in an automotive piston liner pairing, as detailed in [16]. The reciprocating rig's main structural features are presented in detail in Fig. 1. This comprises a linear contact between a stationary convex AISI 52100 steel pad and a fused silica plate that slides along two linear bearings with a controlled amplitude of $28.6 \mathrm{~mm}$ and nearly sinusoidal velocity profile by means of a cam mechanism. Dead weights are attached to the silica pad specimen holder in order to generate an operating normal load, while a system composed of a heating circulator and two pumps allows the oil to be supplied directly to either side of the contact at a precisely controlled temperature and flow rate.

A high sensitivity load cell is located in direct contact with the steel pad holder. This holder was designed to allow the self-alignment of the steel pad against the fused silica counter-specimen while having a flexible base to facilitate load cell measurements. The friction force signal from the load cell is digitised and stored using LabView software.

A rotary position encoder fixed to the cam mechanisms' shaft is used to measure reciprocating velocity and crank angular position. The correspondence between friction force and crank angular position was achieved by a custom-built 9 bit trigger system that synchronised measurement signals. This permits the simultaneous analysis of boundary friction at the reversal point and corresponding wear track position, with a precision of 0.7 degrees of crankshaft revolution.

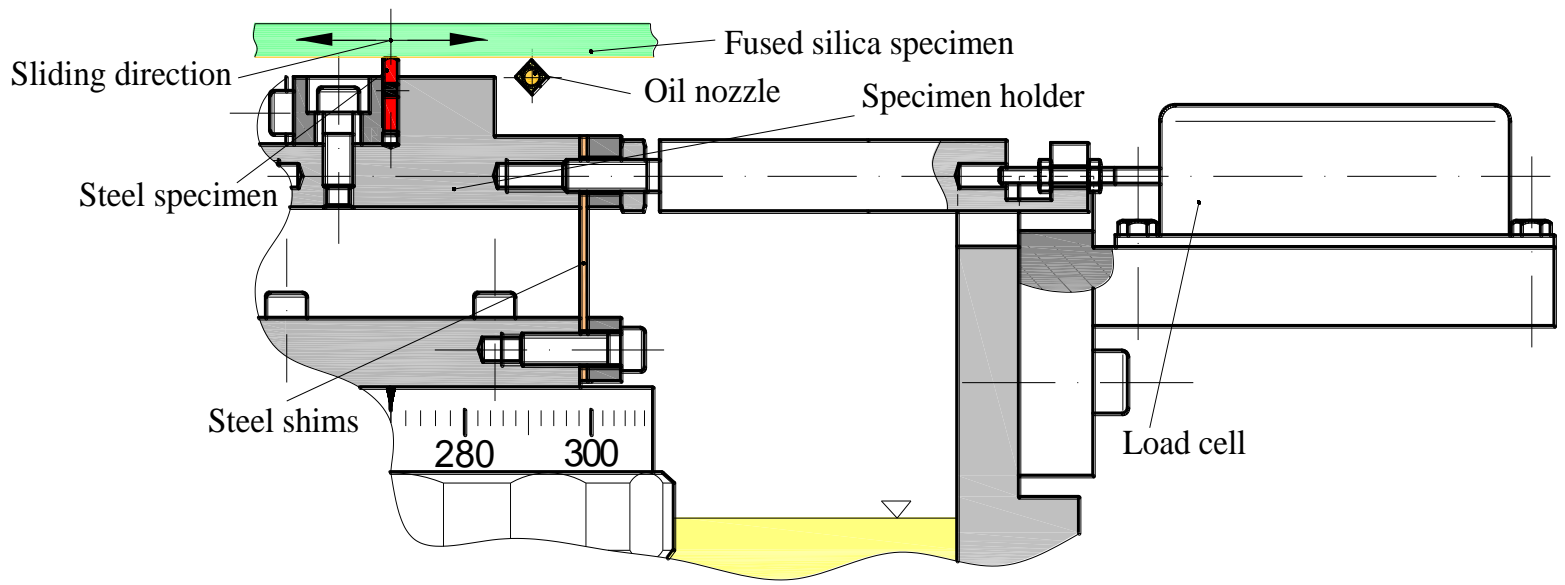

Fig. 1 Reciprocating test rig developed in-house

The reliability of test results obtained using this configuration for both textured and non-textured surfaces has been confirmed through extensive repeat tests under different sets of conditions.

\subsection{Test Parameters and Specimens}

HPFS Standard Grade fused silica rectangular pads with dimensions of $125 \times 15 \times 5 \mathrm{~mm}$ were used as the reciprocating specimens in this study. Textured patterns, consisting in linear grooves orientated normal to the direction of sliding, were applied to these specimens. There are a number of 
differences, between the materials used in this study and those found in commercial piston liners, where surfaces are typically cast-iron or aluminium with a typical roughness of $0.2-0.3 \mu \mathrm{m}$ that results from honing. Furthermore, the use of coating such as DLC are increasing used to coat automotive liners. As discussed in Section 3, this means that the wear mechanism in these experiments differ from those occurring in current piston liner interfaces. However, the model contact between silica and steel was deliberately chosen in order to replicate the exact conditions used in three previous studies [refs]. This enables us to correlate our observations with previously established understanding of how texture induced friction reduction operates (it was important that we tested conditions where laser texture has a proven effect of tribological performance). An additional reason for using silica specimens is their uniform nature and surface finish $(12 \mathrm{~nm})$. This has enabled us to measure and characterise the wear behaviour accurately and to remove the influence of initial surface roughness on texture behaviour.

The pockets were produced using Laser Surface Texturing (LST) [4]. Also known as laser honing, this method employs an ultra-fast picosecond laser which emits optical pulses with a duration of $10 \mathrm{ps,}$ frequency of $10 \mathrm{kHz}$ and wavelength of $355 \mathrm{~nm}$, and power of 5 micro joules. Such short pulse durations are vital in order to ablate micron sized portions from a surface before the material has time to undergo significant thermal changes. The resulting geometric uniformity is necessary since we have found that pockets with micron sized ridges, or with walls that are few degrees off perpendicular, result in significant underperformance. 


\begin{tabular}{|c|c|c|c|c|c|}
\hline \multirow[b]{2}{*}{$\begin{array}{c}\text { Specimen } \\
\text { No. }\end{array}$} & \multicolumn{3}{|c|}{ Surface Texture Geometry } & \multirow[b]{2}{*}{ Image } & \multirow[b]{2}{*}{ Sample name } \\
\hline & $\begin{array}{c}\text { Breadth } \\
{[\mu \mathrm{m}]}\end{array}$ & $\begin{array}{l}\text { Depth } \\
{[\mu \mathrm{m}]}\end{array}$ & $\begin{array}{c}\text { Separation } \\
{[\mu \mathrm{m}]}\end{array}$ & & \\
\hline 1 & 40 & 7 & 500 & & $40 \times 7 \times 500$ \\
\hline 2 & 80 & 3 & 500 & & $80 \times 3 \times 500$ \\
\hline 3 & 80 & 3 & 1000 & & $80 \times 3 \times 1000$ \\
\hline 4 & 300 & 7 & 1100 & & $300 \times 7 \times 1100$ \\
\hline
\end{tabular}

The orientation of the pockets was selected based on previous experimental studies [16], which showed that grooves perpendicular to the direction of sliding are most effective in reducing friction under both mixed and boundary lubricated conditions. It is also important that pockets can become entirely trapped within the contact zone, since these perform better than those with pockets that are longer than the contact zone in the direction of travel $[15,16,19]$. In this study, three different pockets breadths were selected; firstly, two breadths of $40 \mu \mathrm{m}$ and $80 \mu \mathrm{m}$, which are small enough to be completely enclosed within the contact area and secondly, a pocket breadth of $300 \mu \mathrm{m}$ which is much larger than the elastic contact width. Table 1 summarises the geometries of the selected patterns together with the corresponding images, while Figure 2 shows an example of an interference image of the contact with an $80 \mu \mathrm{m}$ breadth pocket close to the inlet of the contact (obtained by viewing the contact through transparent silica specimen with an optical microscope).

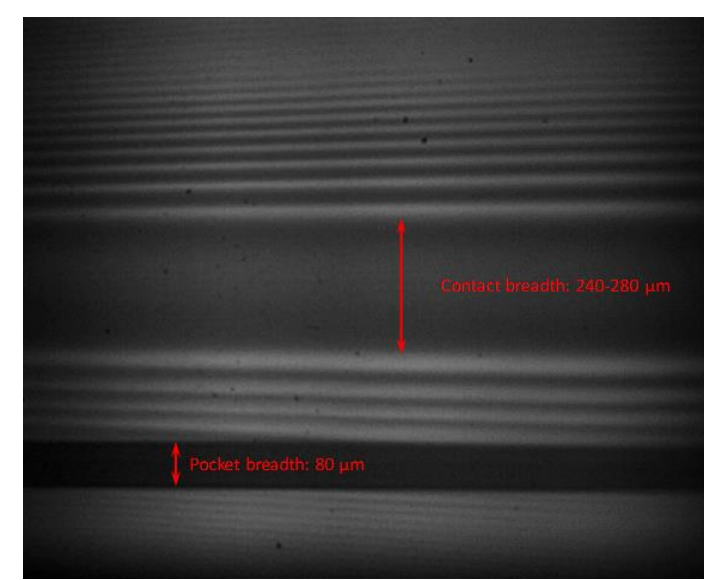

Fig. 2 Interference image of the contact showing a pocket close to the inlet 
The counterpart specimens - all identical AISI 52100 steel pads replicating the top piston ring -, are $10 \mathrm{~mm}$ long and curved in the direction of sliding resulting in a convergent-divergent form. Following a grinding process of the initial fully hardened sample, a minimum stock of $0.15 \mathrm{~mm}$ was removed. The $2 \mathrm{~mm}$ width side of the specimen was subsequently ground at a $40 \mathrm{~mm}$ radius using a dedicated jig, which allows for accurate polishing along the entire length of the pad without modifying the radius. The convex surface was mirror polished according to the roughness presented in Fig. 3.

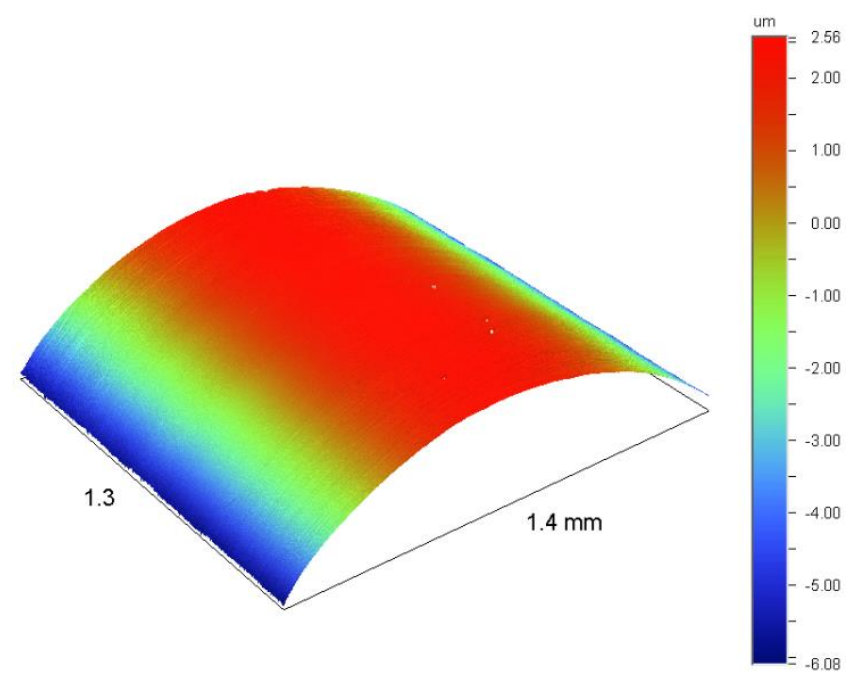

Fig. 3 Three dimensional surface profile of a mirror-polished steel specimen

In all friction force and wear tests, a normal load of $155 \mathrm{~N}$ was applied and a crank angular velocity of $1 \mathrm{~Hz}$ was maintained. Such high load, low speed, conditions were chosen in order to minimise lubricant entrainment, increasing surface contact and wear. Wear was also promoted by using an additive-free mineral oil, which was continuously supplied to the contact region while accurately controlling its temperature, and consequently viscosity, throughout the entire testing session. The lubricant properties alongside all other test parameters are listed in Table 2.

Table 2 Experimental conditions. (note: the quoted lambda value is that for the non-texture specimen, calculated from the mid-stroke film thickness at the start of the test. As the test progress and wear takes place, the lambda value will increase due to roughening of the specimens).

\begin{tabular}{|c|c|c|c|c|c|c|c|}
\hline \multirow[b]{2}{*}{$\begin{array}{l}\text { Load, } \\
\text { W [N] }\end{array}$} & \multirow[b]{2}{*}{$\begin{array}{c}\text { Crank } \\
\text { angular } \\
\text { velocity, } \\
{[\mathrm{Hz}]}\end{array}$} & \multirow[b]{2}{*}{$\begin{array}{c}\text { Oil } \\
\text { Temperature } \\
{\left[{ }^{\circ} \mathrm{C}\right]}\end{array}$} & \multicolumn{3}{|c|}{ Oil properties } & \multirow[b]{2}{*}{$\begin{array}{c}\text { Time length } \\
\text { of the test, } t \\
{[h]}\end{array}$} & \multirow[b]{2}{*}{$\begin{array}{c}\text { Initial } \\
\text { Lambda } \\
\text { Ratio, } \\
\Lambda[-]\end{array}$} \\
\hline & & & $\begin{array}{c}\text { Dynamic } \\
\text { viscosity, } \eta \\
\text { [mPa s] }\end{array}$ & $\begin{array}{c}\text { Kinematic } \\
\text { viscosity, v } \\
{\left[\mathrm{mm}^{2} / \mathrm{s}\right]}\end{array}$ & $\begin{array}{c}\text { Density, } \rho \\
{\left[\mathrm{g} / \mathrm{cm}^{3}\right]}\end{array}$ & & \\
\hline 155 & 1 & 80 & 18.313 & 22.947 & 0.798 & 4 & 2.65 \\
\hline
\end{tabular}

\subsection{Experimental Protocol}


Precise calibrations of the isometric load cell were performed both before and after each test was carried out. To do this, various known weights were attached to the steel pad holder in order to displace it in both tangential directions (tension and compression) and thus define the relationship between load and voltage. During testing, the average of these two was used to convert voltage output into friction force.

The fused silica and the steel specimens were consecutively sonicated in toluene and isopropanol before all traces of solvent were dried out using a heat gun. Wear tests, each with a duration of four hours were carried out on all the textured specimens presented in Table 1 as well as the non-textured reference. During each test, the friction force variation for one complete stroke was recorded after one minute and after three minutes, then every five minutes for the first half hour and every ten minutes for the remaining three and a half hours. At the end of the test, the cleaning procedure previously described was repeated and the topography of wear track around the reversal point was recorded using the Veeco Wyco NT9100 optical profiler. The Veeco's area of analysis was a rectangle, $2 \mathrm{~mm}$ wide by $3.5 \mathrm{~mm}$ long. This rectangle was located such that, at reversal, its short axis coincided with that of the elliptical contact. In this way the region of highest wear fell within its borders. In the direction of sliding, the rectangle's $2 \mathrm{~mm}$ width extend outside the wear track, thus encompassing a non-worn region, which could provide a datum from which the wear volume could be calculated.

\section{Results and discussion}

Many of the studies into the impact of surface texture on wear and frictional response have been conducted on ball-on-plate contact configurations using commercially available pin-on-disc tribometers $[18,19,42,43]$. These test conditions differ significantly from those found in an actual piston ringcylinder liner pairing. The test apparatus developed specifically for the present research allows for the friction force variation on every stroke to be plotted as a function of crank angle throughout the wear test. This provides more detailed information than the previously obtained single point measurements. A graphically depicted comparison of the frictional response was thus possible at any time during the test. Figure 4 displays the friction force variation, captured for all four textured specimens together with the smooth reference, plotted as a function of crank angle, throughout the entire wear test.

Over the first few cycles of each test $\left(t_{0}\right)$, the smooth pad exhibits the lowest friction force for the majority of the stroke compared with any of the textured specimens. This is explained as follows. At the start of the test, negligible wear has occurred on any of the sliding pairs and the composite roughness was approximately equal to its initial value of $12 \mathrm{~nm}$. For a mid-stroke film thickness of around $38 \mathrm{~nm}$ (calculated by applying Dowson and Higginson's Equation [44] for the non-textured pairing), this roughness gives nominal lambda values in the region of 2.65 , showing the bearing contacts are operating in the full film hydrodynamic lubrication regime. This initial predominance of hydrodynamic lubrication is further demonstrated by the flat-bottomed "U" shape of the non-textured friction curve; when considering the sinusoidal nature of the varying entrainment speed, this shape must arise from the contact spending the majority of each reciprocation around the lowest point of the Stribeck Curve. As previously shown in $[14,16]$, it is under these full film lubrication conditions, that surface texture can have the effect of increasing friction, and hence explains why the non-textured specimen performs the best, initially. 


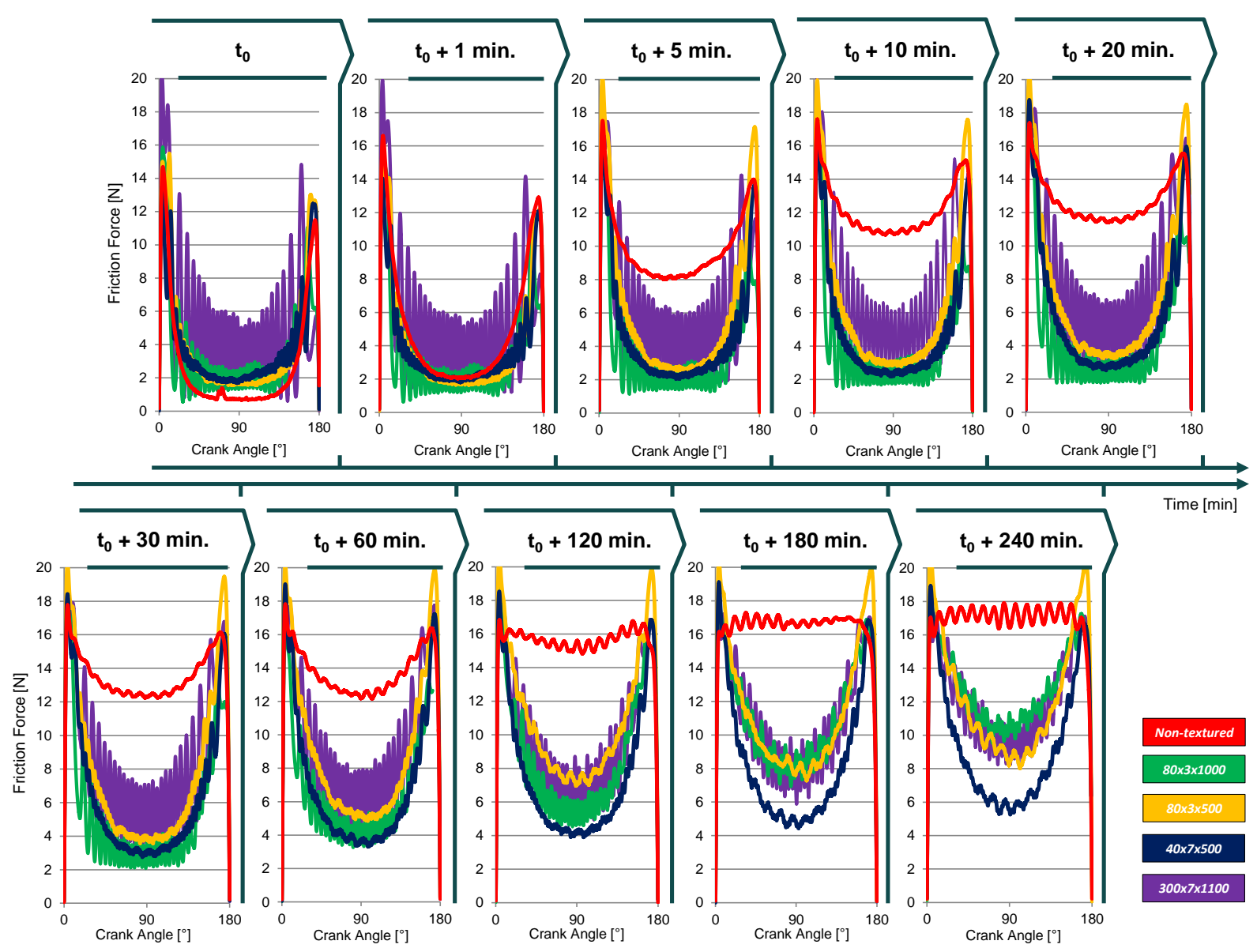

Fig. 4 Friction force comparison between all textured specimen and the smooth reference over the four hour wear tests.

After approximately 5 minutes, and as soon as the first scars of wear appear on its surface, the nontextured reference begins to show the highest friction compared to all of the other textured specimens. This is because the appearance of wear signifies the transition of the contact from full film to mixed lubrication conditions due to an increase in composite surface roughness that is greater than the lubricant film thickness. It is under this regime that surface texture is most beneficial in terms of reducing friction, as proven by numerous experiments $[14,16,17,45]$. No further changes in ranking were recorded over the remaining four hours test, with the non-textured specimen continuing to exhibit the highest friction at every time step. Moreover, after approximately three hours of rubbing $\left(\mathrm{t}_{0}+180 \mathrm{~min}\right.$.), the nontextured bearing reaches the boundary regime, exemplified by a constant friction force along the entire stroke (i.e. the wear has advance to such an extent that the increasing hydrodynamic separation as the bearing accelerates towards mid-stoke is insignificant compared to the roughness).

Further inspection reveals that the most sparsely textured specimen $(80 \times 3 \times 1000)$ displays the lowest friction force at the beginning of the testing sequence and the highest friction force by the end of the fourth hour, when compared with the other pocketed specimens. Conversely, the deepest and most dense pattern $(40 \times 7 \times 500)$ displays the lowest friction from the first hour of the test onwards. This behaviour suggests that deeper and more densely packed pockets are desired towards the end of the stroke (i.e. in the boundary and mixed lubrication regime), whereas sparser and more shallow pockets are needed towards the middle of the stroke (i.e. in transition between mixed to full film regime), and surface pockets should in fact be avoided when the bearing is operating in full film lubrication regime. 
For a better visualisation of the above mentioned trends, the mid-stroke friction force, captured between $60^{\circ}$ and $120^{\circ}$ crank angle, has been averaged and is plotted as a coefficient of friction against sliding time in Figure 5. Here, significant and constant reductions of up to $70 \%$ are evident over the last two hours of tests when comparing the best performing textured pattern $(40 \times 7 \times 500)$ against the nontextured reference.

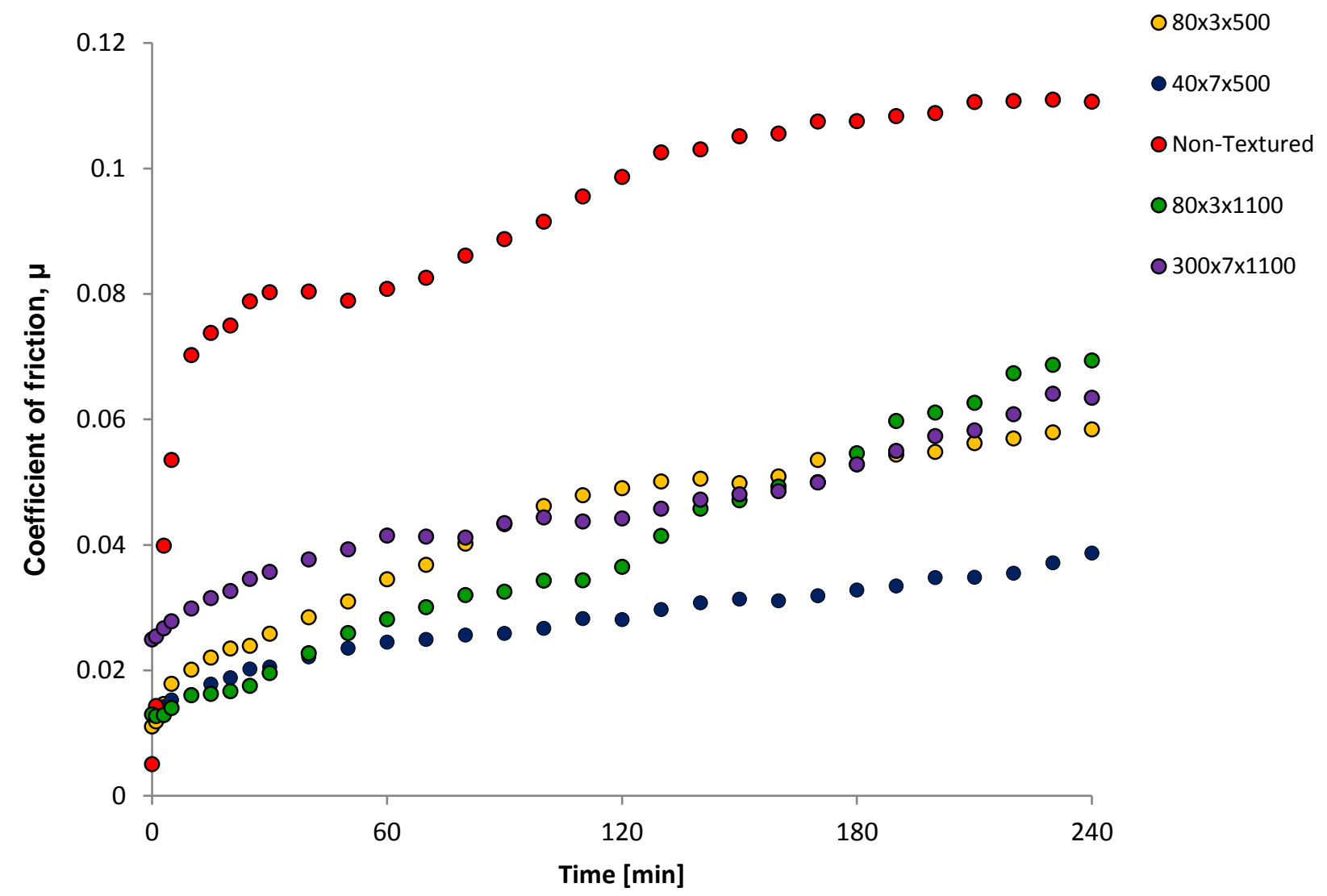

Fig. 5 Variation of coefficient of friction (average values obtained between crank angles of $60^{\circ}$ and $120^{\circ}$ ) with sliding time for all textured and non-textured fused silica specimens

Figure 6 shows the data from Figure 5 replotted in terms of percentage improvement for the four textured bearings compared with the non-textured reference. Here, the relative change in performance between the non-textured and texture specimens can be clearly observed. In addition to this, it can be seen that the pattern consisting of pockets wider than the contact in the direction of travel $(300 \times 7 \times 1100)$ exhibits slightly higher friction than the other texture geometries over the initial stage of the test (i.e. during the first hour). This requirement that pocket should be less wide that the contact in order to provide greatest friction reduction is consistent with previous findings for texture specimens. However, it should also be noted that by the end of the test, there are no discernable differences between the friction performances of the $300 \mu \mathrm{m}$ breadth pockets and the $80 \mu \mathrm{m}$ breadth ones. 


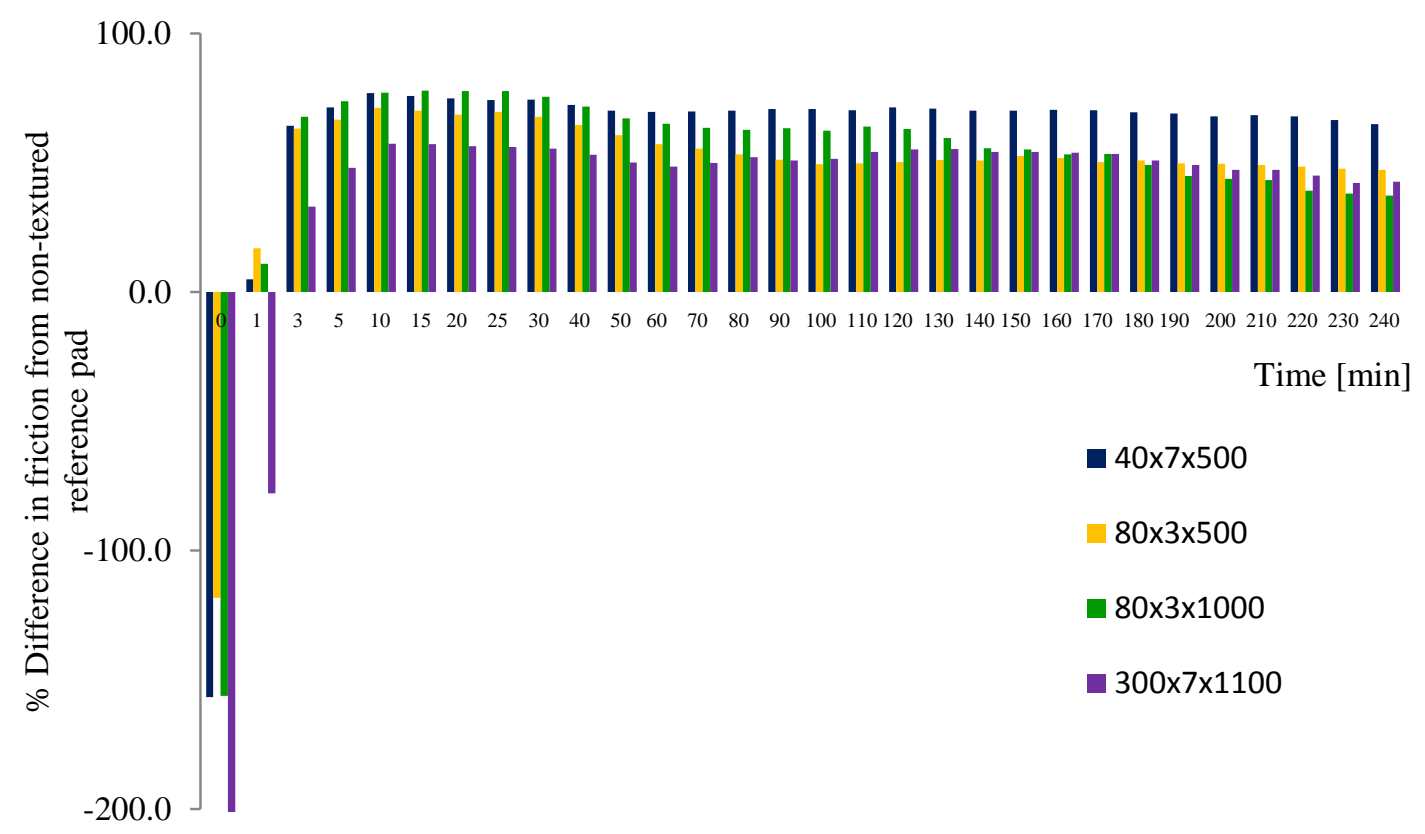

Fig. 6 Percentage difference in friction between each textured specimen and the non-textured reference.

Another key observation from Figure 4 is the presence of significant peaks in the friction traces from certain pocket configurations. Rather than being experimental noise, these peaks correspond to lines of pockets entering the contact. Upon reaching a line of pockets, the contact area reduces considerably (by a factor of 10, which, under mixed lubrication regime has a significant impact on friction force) and, as a consequence, a local spike in friction is observed. As soon as each pocket leaves the contact, the film thickness increases and friction falls below the initial level. This effect is more pronounced for the textured specimens with a more sparse texture pattern, i.e. $300 \times 7 \times 1100$ and $80 \times 3 \times 1000$. As the frequency with which pockets enter the contact doubles (i.e. with specimens $40 \times 7 \times 500$ and $80 \times 3 \times 500$ ), the observation of this local friction effect becomes difficult. This relationship between friction force and the frequency with which textured features pass through a contact is an important topic which will be investigated in future research. 
For a better visualisation of this transient effect, the friction force variation of the $80 \times 3 \times 500$ textured pad at $t_{0}+1 \mathrm{~min}$ was plotted alongside the non-textured reference as a function of distance travelled along the reciprocating stroke in Fig. 7. It can be observed that, although at the beginning of the stroke both pads exhibit similar friction, the first pocket placed immediately after reversal results in a considerable reduction of frictional response.

The friction force shows a temporary increase as each line of pockets enters the contact, however, as soon as the micro-features leave the contact, the friction force drops significantly compared with the nontextured pad. This behaviour is consistent with a recent study into the transient effects of surface texture [32]. Furthermore, since it is now known that the overall reduction in friction that results from multiple pocket entrainments is due to an increase in film thickness [31], it seems reasonable to assume that the observed transient variation in friction force is due to a corresponding variation in film thickness, caused by the entrainment of pockets. This assumption is supported by the fact that, in Figure 7, the optical profile image shows a regions of wear that are localised around the edges of the pockets. The question remains as to exactly what mechanism is responsible for the film thickness increase as each pocket leaves the contact and so far our experiments have not been able to prove or disprove inlet suction, the Trip effect, or some other hydrodynamic mechanism as being responsible.

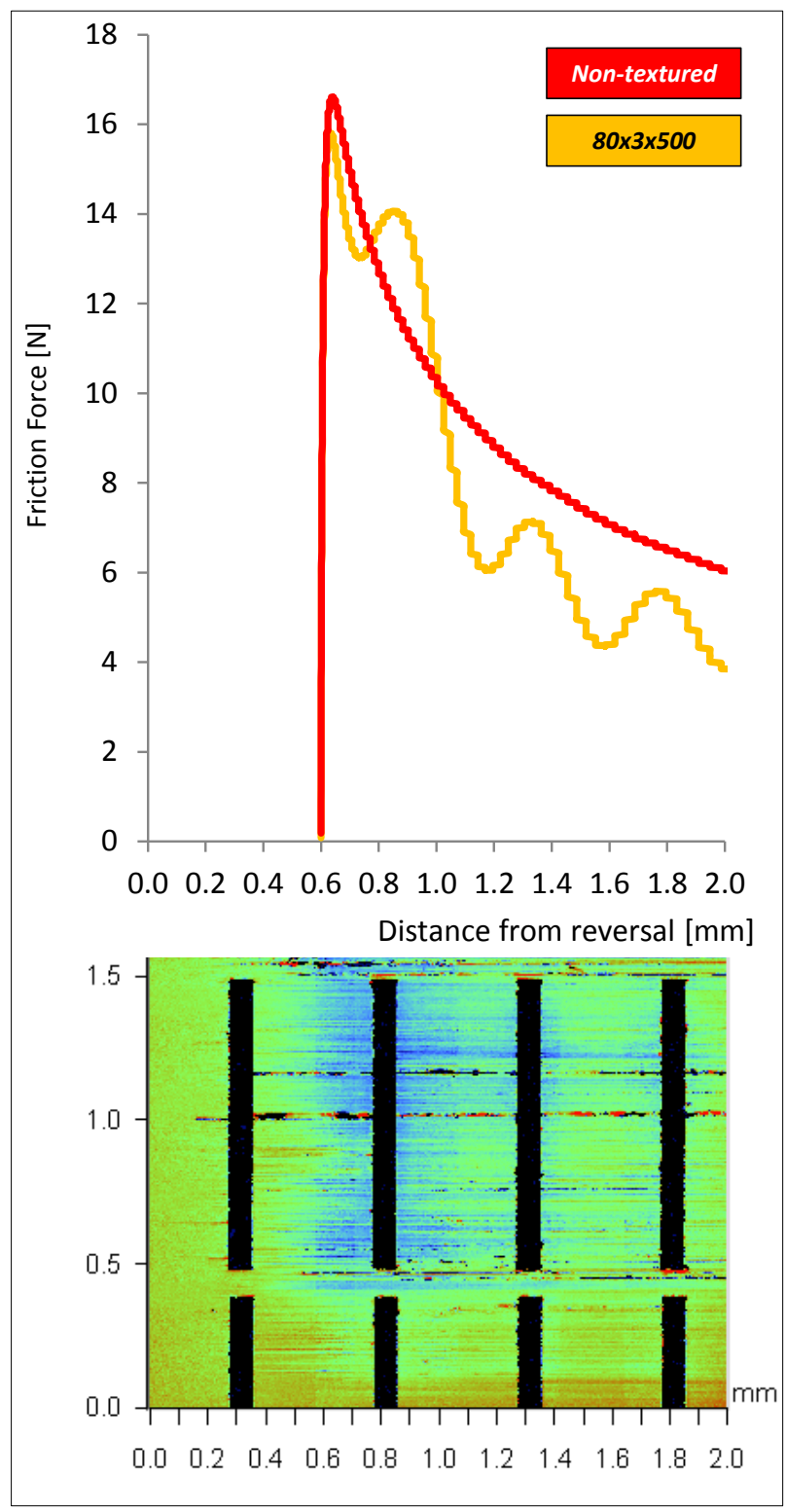

Fig. 7 Friction force and wear topography variation vs. sliding distance at the end of travel

The counterpart steel specimens were analysed by Veeco Wyko surface profiler and no differences in average mean roughness greater than $5 \mathrm{~nm}$ were detected when comparing the five steel specimens. To exemplify this, Fig. 8 illustrates the surface topography of the steel pads before a test (a), after the four hours long wear test against a non-textured fused silica pad (b), and at the end of the test employing the pocketed specimen $40 \times 7 \times 500$. Most probably, this low wear behaviour of the steel pads is due to the hardening process that the specimens underwent prior testing. For this reason, this study focusses on the wear observed on the silica liner specimen, and wearing of the steel ring specimen will be the subject of further testing. 
a)
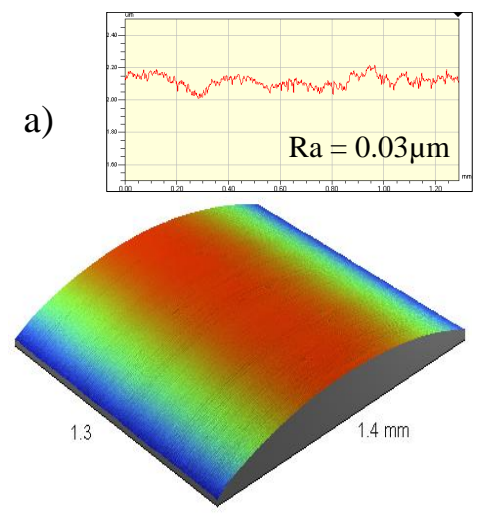

b)
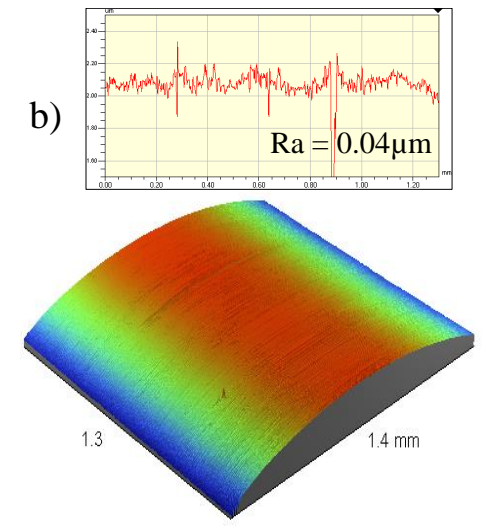

c)
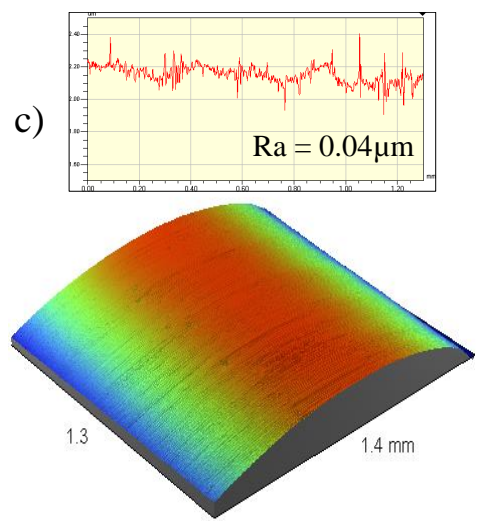

Fig. 8: Three dimensional surface topography of the steel specimens used for the wear study: a) Surface plot captured before the test against the non-textured counterpart; b) Surface plot captured after the test against the non-textured counterpart; c) Surface plot captured after the test against the 40 x 7 x 500 counterpart;

Two-dimensional images of the typical wear tracks on the fused silica pads at the reversal point are shown in Figure 9. The $3.5 \times 2 \mathrm{~mm}$ images were recorded, for all five tested specimens, at the same point along the stroke. This enabled accurate comparisons of the wear tracks and corresponding wear volumes. The first $0.2 \mathrm{~mm}$ slice on the horizontal axis, outside of the contacting region was considered the initial, unworn, level and was set as the datum surface before performing the wear measurements. Similar to IC engines and other applications comprising reciprocating bearings, wear occurred almost exclusively towards the end of the strokes due to a significant reduction in speed and hence lubricant entrainment in these regions. Indeed, optical profiles of the worn surfaces indicate that the greatest wear scar depths on the fused silica specimens occurred in all cases on the first $1.5 \mathrm{~mm}$ of the reciprocating stroke, immediately after reversal. Although Figure 9 presents topography for only one end of stroke, a near identical wear pattern was observed at the other end.

Despite similar wear scar dimensions in the direction of sliding (approx. $1.5 \mathrm{~mm}$ ) for all the specimens, a qualitative and quantitative analysis of the volume of wear removed from the $3.5 \mathrm{~mm}^{2}$ area around reversal, as indicated in Fig. 9, was performed. This shows the highest volume of wear for the non-textured specimen, followed by the $80 \times 3 \times 1000$ sample, whereas the $300 \times 7 \times 1100$ pattern proved most resilient to wear. This ranking of specimen performance leads us to conclude that there is a close connection between the volume of wear removed from the surface and the volume of oil each pattern brings to the contact during one stroke. Figure 10 presents this conclusion graphically: for each specimen, the volume of each pocket multiplied by the number of pockets replenishing the contact with each stroke is shown on the left, while the graph on the right displays the volume of wear removed over the four hour test. 

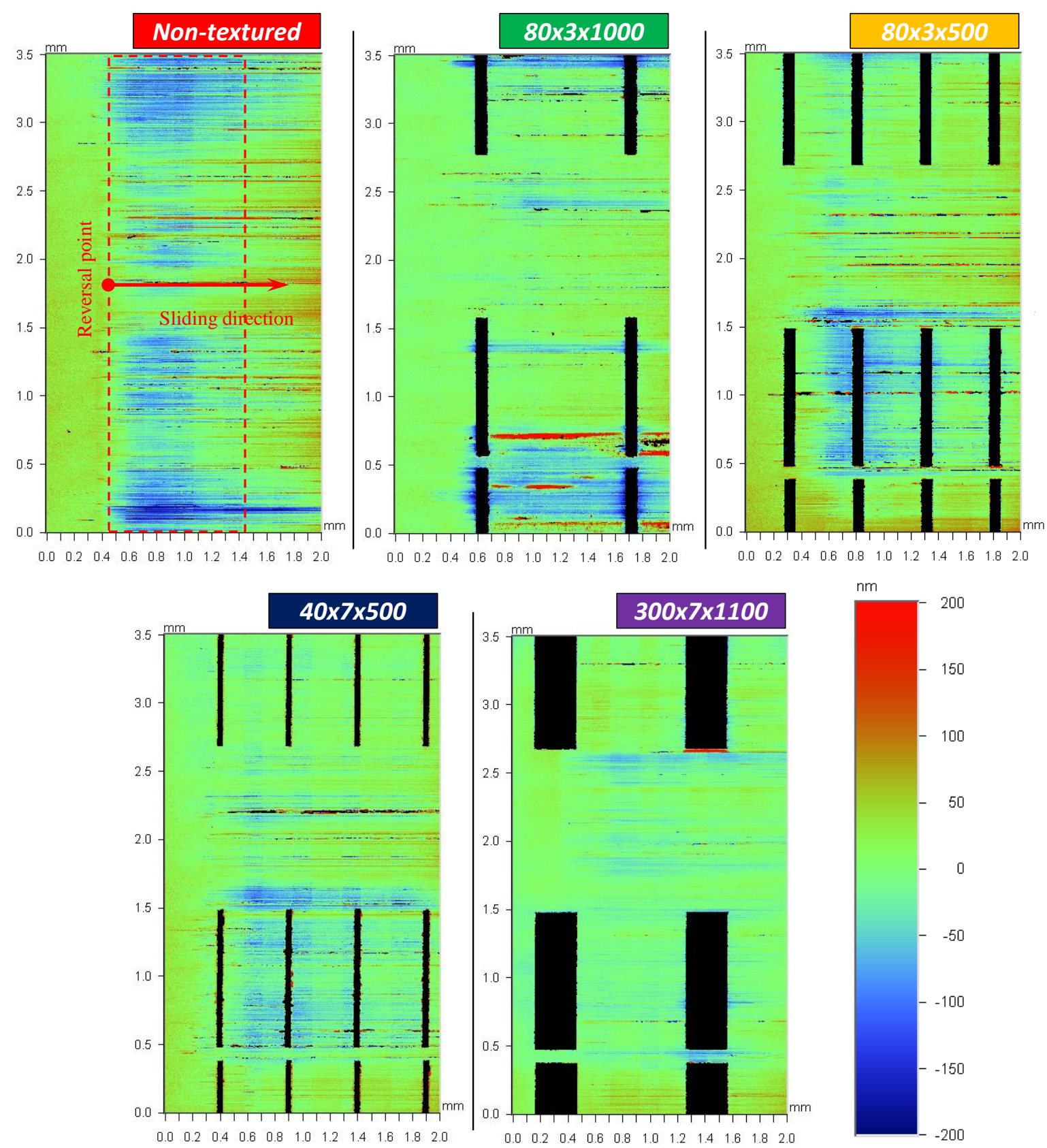

Fig. 9 Two dimensional surface topography plots of all tested specimens as obtained post-test by the Veeco Wyco optical profiler

It is immediately apparent from Figure 10 that, as the volume of oil brought into the contact increases from one pattern to the next, the volume of wear observed throughout the test decreases in perfect correlation. The non-textured specimen, with no pockets to replenish the contact area, exhibits the highest wear volume when compared with any of the textured surfaces. The $80 \times 3 \times 1000$ pattern on the other hand shows a higher volume of wear removed from the surface when compared with a twice as dense pattern, $80 \times 3 \times 500$, which in turn shows lower wear performance when compared with the much deeper $40 \times 7 \times 500$ pattern. Finally, the $300 \times 7 \times 1100$ textured pattern, which replenishes the contact with a volume of oil at least three times higher than any of the other specimens, exhibited the lowest wear volume throughout the test. It should be noted that this monotonic decrease would not have been observed if wear had been plotted against pocket length, depth or width individually. 

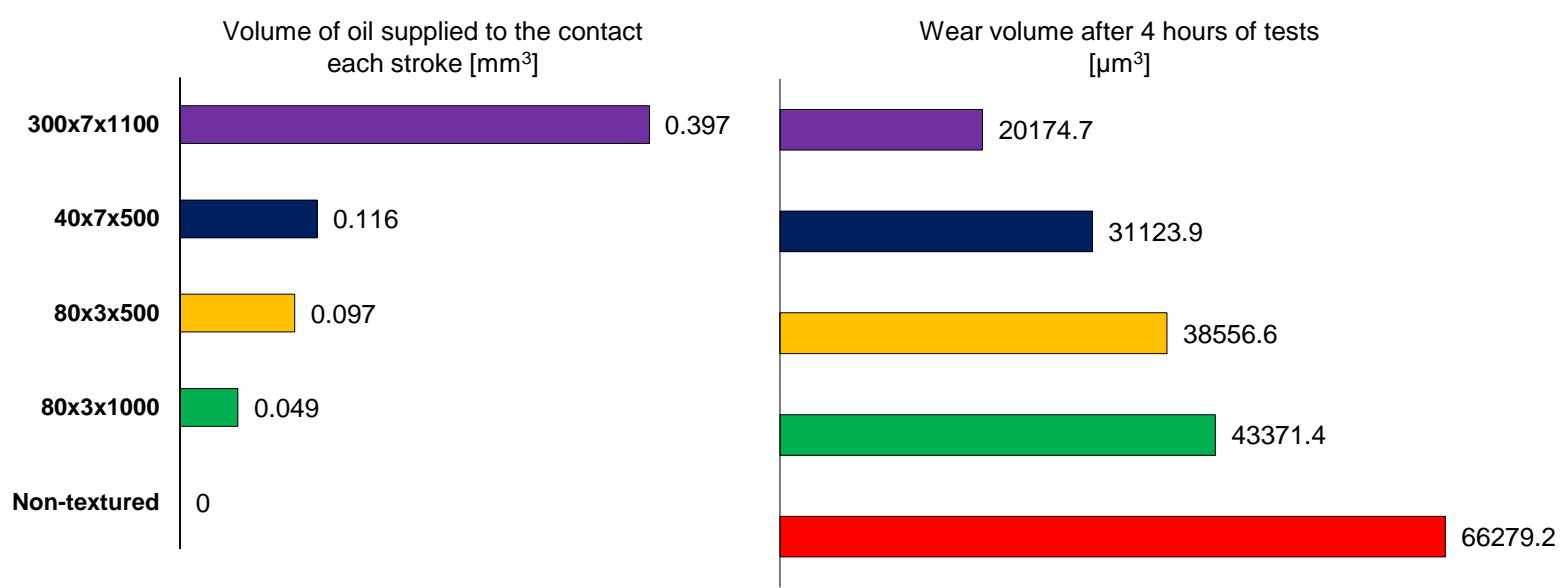

Fig. 10 Correlation between the volume of oil supplied to the contact by the textured pattern each stoke and the wear behaviour

To show the link between wear and friction behaviour, Figure 11 plots average friction coefficient over the last 60 minutes of testing alongside final wear volume for different pocket entrainment volumes. Here, a positive correlation can be observed for four out of five specimens - specifically, a lower average friction corresponds to a lower final wear volume. This correlation may at first seem to be due to the fact that pockets cause an increase in film thickness [31], and that this causes a reduction in both friction (as is known to be the case [16,32]), and also wear. However an exception to this trend is the $300 \times 7 \times 1100$ pocketed specimen, which showed the lowest wear of all specimens but higher friction than denser pattern samples $40 \times 7 \times 500$ and $80 \times 3 \times 500$. It is important to note that this exception can be attributed to the $300 \mu \mathrm{m}$ width of the high friction pockets, which are larger than the $240 \mu \mathrm{m}$ long contact. Such wide pockets are known to allow oil to flow from the contact and hence cause a collapse of the film [16]. This is an important result and seems to suggest that pockets reduce wear and friction via different mechanisms. 


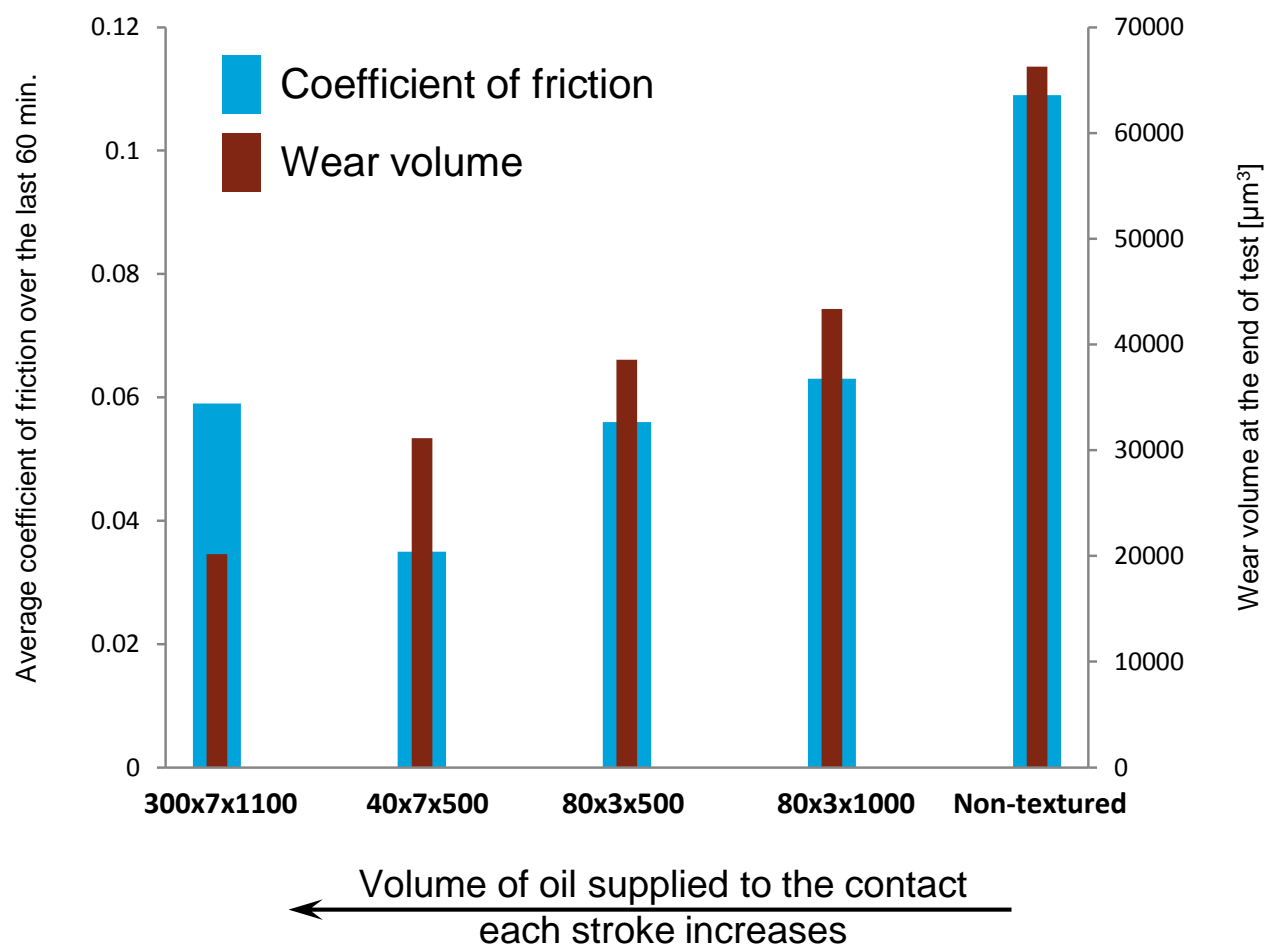

Fig. 11 Correlation between the average coefficient of friction calculated over the last 60 minutes of testing and the final wear volume

It may be that the additional mechanism with which pockets reduce wear is that of debris trapping. It is also possible that pockets are reducing wear by replenishing lubricant and reducing preventing starvation, however this mechanism would not explain the discrepancy between wear and friction behaviour. Furthermore, it should be noted that the excess oil was supplied to the contact inlet by means of the lubricant pumps, so that starvation is only likely to result from the ingestion of the cavitated region following reversal.

It should be noted that the fused silica pads used in this study will wear differently to metal specimens, however, they were selected so that results presented here can be correlated with film thickness and friction results obtained in previous studies [16,31,32]. Several studies have looked at the evolution of piston/liner wear in using more representative materials [Priest] and the focus of our ongoing work is to extend this understanding to laser textured surfaces by testing rougher, metal-metal contacts using the methods described in this paper.

\section{Conclusions}

A specially developed test apparatus was employed to assess the effects of laser produced surface texture upon friction and wear behaviour. This involved reciprocating sliding tests on a linear contact for a duration of four hours. Various patterns comprising pockets orientated normal to the direction of sliding were compared against a non-textured reference specimen, and this lead to the following key conclusions: 
- For unworn contacts with high lambda values, such as those found at the start of testing, the presence of texture causes an increase in friction compared to the non-textured reference case (this agrees with previous research on full film conditions).

- As specimens become worn and the contact enters the mixed lubrication regime, surface texture acts to significantly reduce the friction coefficient. Under these conditions, friction reductions of up to $70 \%$ were observed when comparing the best performing microtextured pattern $(40 \times 7 \times 500)$ against the non-textured reference. This suggests that surface texture performance is not only effective on mirror polished laboratory specimens, but is likely to work on actual machine components where it will limit the deleterious effects of wear on friction.

- For the rectangular pockets tested, both friction and wear reduce monotonically as the sum of the pocket volumes along the stroke increases. This may aid texture design, since it means that individual pocket width and depth values can largely be ignored, so long as the overall volume is maximised. The only exception to this trend is when the pockets are larger than the contact area. In this case, friction increases due to a collapse of the lubricant film, while wear reduction remains unaffected and may suggest that pockets reduce wear and friction via separate mechanisms. This supports the theory that, in addition to providing a hydrodynamic film thickness boost, pockets act as reservoirs, which reduce starvation by increasing the supply of lubricant to the contact - particularly after reversal when the cavitated outlet becomes the inlet.

- However, when textured patterns consist of pocket that are too wide,(compared to the contact width) and too sparsely distributed (for a given sliding speed) they act to locally increase the friction force as they pass through contact, generating local spikes of higher friction. This is due to the significant reduction of the contact area as a line of features enters the contact.

- Considering wear reduction when selecting the optimal pattern is thus an important step but must be complemented with thorough analysis of the frequency at which pockets enter the contact, the relative size of the pockets compared to the contact, and the precise pocket location relative to after reversal point.

\section{Acknowledgement}

The authors are grateful to Khizer Tufail and Arup Gangopadhyay for their advice and support. The project was funded by the Ford Motor Company.

\section{References}

[1] D.B. Hamilton, J.A. Walowit, C.M. Allen, A Theory of Lubrication by Micro- irregularities, Trans. ASME J. Basic Eng. 88 (1966) 177-185.

[2] K. Holmberg, P. Andersson, A. Erdemir, Global energy consumption due to friction in passenger cars, Tribol. Int. 47 (2012) 221-234. doi:10.1016/j.triboint.2011.11.022.

[3] European Parliament, Regulation (EC) no 443/2009 setting emission performance standards for new passenger cars as part of the Community's integrated approach to reduce $\mathrm{CO} 2$ emissions from light-duty 
vehicles, Off. J. Eur. Union. (2009) 1-15.

[4] H. Costa, I. Hutchings, Some innovative surface texturing techniques for tribological purposes, Proc. Inst. Mech. Eng. Part J J. Eng. Tribol. (2014). doi:10.1177/1350650114539936.

[5] A. Ronen, I. Etsion, Y. Kligerman, Friction-Reducing Surface-Texturing in Reciprocating Automotive Components, Tribol. Trans. 44 (2001) 359-366. doi:10.1080/10402000108982468.

[6] I. Etsion, State of the Art in Laser Surface Texturing, J. Tribol. 127 (2005) 248. doi:10.1115/1.1828070.

[7] G. Ryk, Y. Kligerman, I. Etsion, Experimental Investigation of Laser Surface Texturing for Reciprocating Automotive Components, Tribol. Trans. 45 (2002) 444-449.

[8] P. Brajdic-Mitidieri, A.D. Gosman, E. Ioannides, H.A. Spikes, CFD Analysis of a Low Friction Pocketed Pad Bearing, J. Tribol. 127 (2005) 803-812. doi:10.1115/1.2032990.

[9] M.T. Fowell, S. Medina, A.V. Olver, H.A. Spikes, I.G. Pegg, Parametric study of texturing in convergent bearings, Tribol. Int. 52 (2012) 7-16. doi:10.1016/j.triboint.2012.02.013.

[10] S. Medina, M.T. Fowell, S.-C. Vlădescu, T. Reddyhoff, I. Pegg, a. V. Olver, et al., Transient effects in lubricated textured bearings, Proc. Inst. Mech. Eng. Part J J. Eng. Tribol. 0 (2015) 1-15. doi:10.1177/1350650115572448.

[11] I. Krupka, M. Hartl, M. Zimmerman, P. Houska, S. Jang, Effect of surface texturing on elastohydrodynamically lubricated contact under transient speed conditions, Tribol. Int. 44 (2011) 11441150. doi:10.1016/j.triboint.2011.05.005.

[12] L. Mourier, D. Mazuyer, F.-P. Ninove, A.A. Lubrecht, Lubrication mechanisms with laser-surfacetextured surfaces in elastohydrodynamic regime, Proc. Inst. Mech. Eng. Part J J. Eng. Tribol. 224 (2010) 697-711. doi:10.1243/13506501JET771.

[13] M. Scaraggi, F.P. Mezzapesa, G. Carbone, A. Ancona, L. Tricarico, Friction Properties of Lubricated Laser-MicroTextured-Surfaces: An Experimental Study from Boundary- to Hydrodynamic-Lubrication, Tribol. Lett. 49 (2012) 117-125. doi:10.1007/s11249-012-0045-2.

[14] A. Kovalchenko, O. Ajayi, A. Erdemir, G. Fenske, I. Etsion, The Effect of Laser Surface Texturing on Transitions in Lubrication Regimes During Unidirectional Sliding Contact, Tribol. Int. 38 (2005) 219225. doi:10.1016/j.triboint.2004.08.004.

[15] H.L. Costa, I.M. Hutchings, Hydrodynamic lubrication of textured steel surfaces under reciprocating sliding conditions, Tribol. Int. 40 (2007) 1227-1238. doi:10.1016/j.triboint.2007.01.014.

[16] S.-C. Vlădescu, A. V. Olver, I.G. Pegg, T. Reddyhoff, The effects of surface texture in reciprocating contacts - An experimental study, Tribol. Int. 82 (2015) 28-42. doi:10.1016/j.triboint.2014.09.015.

[17] D. Braun, C. Greiner, J. Schneider, P. Gumbsch, Efficiency of laser surface texturing in the reduction of friction under mixed lubrication, Tribol. Int. 77 (2014) 142-147. doi:10.1016/j.triboint.2014.04.012.

[18] U. Pettersson, S. Jacobson, Influence of surface texture on boundary lubricated sliding contacts, Tribol. 
Int. 36 (2003) 857-864. doi:10.1016/S0301-679X(03)00104-X.

[19] U. Pettersson, S. Jacobson, Friction and Wear Properties of Micro Textured DLC Coated Surfaces in $\begin{array}{llllll}\text { Boundary Lubricated } & \text { Sliding, } & \text { Tribol. } & \text { Lett. } & 17 & \text { (2004) }\end{array}$ doi:10.1023/B:TRIL.0000044504.76164.4e.

[20] H.L. Costa, I.M. Hutchings, Effects of die surface patterning on lubrication in strip drawing, J. Mater. Process. Technol. 209 (2009) 1175-1180. doi:10.1016/j.jmatprotec.2008.03.026.

[21] M. Wakuda, Y. Yamauchi, S. Kanzaki, Y. Yasuda, Effect of surface texturing on friction reduction between ceramic and steel materials under lubricated sliding contact, Wear. 254 (2003) 356-363. doi:10.1016/S0043-1648(03)00004-8.

[22] V. Macián, B. Tormos, V. Bermúdez, L. Ramírez, Assessment of the effect of low viscosity oils usage on a light duty diesel engine fuel consumption in stationary and transient conditions, Tribol. Int. 79 (2014) 132-139. doi:10.1016/j.triboint.2014.06.003.

[23] J.N. Anno, J. a. Walowit, C.M. Allen, Microasperity Lubrication, J. Lubr. Technol. 90 (1968) 351-355. doi:10.1115/1.3601568.

[24] K. Tønder, Dynamics of rough slider bearings : effects of one-sided roughness / waviness, Tribol. Int. 29 (1996) 117-122.

[25] K. Tønder, Hydrodynamic effects of tailored inlet roughnesses: extended theory, Tribol. Int. 37 (2004) 137-142. doi:10.1016/S0301-679X(03)00043-4.

[26] G.E. Morales-Espejel, Elastohydrodynamic Lubrication of Smooth and Rough Surfaces - PhD Thesis, University of Cambridge, 1993.

[27] J.A. Greenwood, G.E. Morales-Espejel, The behaviour of transverse roughness in EHL contacts, Proc. Inst. Mech. Eng. Part J J. Eng. Tribol. 208 (1994) 121-132. doi:10.1243/PIME.

[28] G.E. Morales-Espejel, P.M. Lugt, J. Van Kuilenburg, J.H. Tripp, Effects of Surface Micro-Geometry on the Pressures and Internal Stresses of Pure Rolling EHL Contacts, Tribol. Trans. 46 (2003) 260-272. doi:10.1080/10402000308982625.

[29] A.V. Olver, M.T. Fowell, H.A. Spikes, I.G. Pegg, "Inlet suction", a load support mechanism in nonconvergent, pocketed, hydrodynamic bearings, Proc. Inst. Mech. Eng. Part J J. Eng. Tribol. 220 (2006) 105-108. doi:10.1243/13506501JET168.

[30] M. Fowell, A.V. Olver, A.D. Gosman, H.A. Spikes, I.G. Pegg, Entrainment and Inlet Suction: Two Mechanisms of Hydrodynamic Lubrication in Textured Bearings, J. Tribol. 129 (2007) 336-347. doi:10.1115/1.2540089.

[31] S.-C. Vlădescu, S. Medina, A. V. Olver, I.G. Pegg, T. Reddyhoff, (Forthcoming) Lubricant film thickness and friction force measurements in a laser surface textured reciprocating line contact simulating the piston ring - liner pairing, Tribol. Int. (2016).

[32] S.-C. Vlădescu, S. Medina, A. V. Olver, I.G. Pegg, T. Reddyhoff, (Forthcoming) The transient friction response of a laser textured, reciprocating contact to the entrainment of individual pockets, Tribol. Lett. 
(2016).

[33] Y.P. Chiu, An Analysis and Prediction of Lubricant Film Starvation in Rolling Contact Systems, A S L E Trans. 17 (1974) 22-35. doi:10.1080/05698197408981435.

[34] M.-T. Ma, I. Sherrington, E.H. Smith, Implementation of an algorithm to model the starved lubrication of a piston ring in distorted bores : prediction of oil flow and onset of gas blow-by, Proc. Inst. Mech. Eng. , Part J J. Eng. Tribol. 210 (1996) 29-44. doi:10.1243/PIME.

[35] P.M. Cann, H. a. Spikes, J. Hutchinson, The Development of a Spacer Layer Imaging Method (SLIM) for Mapping Elastohydrodynamic Contacts, Tribol. Trans. 39 (1996) 915-921. doi:10.1080/10402009608983612.

[36] A. Blatter, M. Maillat, S.M. Pimenov, G.A. Shafeev, A.V. Simakin, E.N. Loubnin, Lubricated sliding performance of laser-patterned sapphire, Wear. 232 (1999) 226-230. doi:10.1016/S0043-1648(99)001507.

[37] A. Borghi, E. Gualtieri, D. Marchetto, L. Moretti, S. Valeri, Tribological effects of surface texturing on nitriding steel for high-performance engine applications, Wear. 265 (2008) 1046-1051. doi:10.1016/j.wear.2008.02.011.

[38] I. Křupka, M. Vrbka, M. Hartl, Effect of surface texturing on mixed lubricated non-conformal contacts, Tribol. Int. 41 (2008) 1063-1073. doi:10.1016/j.triboint.2007.11.016.

[39] M. Varenberg, G. Halperin, I. Etsion, Different aspects of the role of wear debris in fretting wear, Wear 252 (2002) 902-910.

[40] K.H. Zum Gahr, M. Mathieu, B. Brylka, Friction control by surface engineering of ceramic sliding pairs in water, Wear. 263 (2007) 920-929. doi:10.1016/j.wear.2006.11.024.

[41] R.I. Taylor, R.C. Coy, Improved fuel efficiency by lubricant design: A review, Proc. Inst. Mech. Eng. Part J J. Eng. Tribol. 214 (2000) 1-15. doi:10.1177/135065010021400101.

[42] A. Kovalchenko, O. Ajayi, A. Erdemir, G. Fenske, Friction and wear behavior of laser textured surface under lubricated initial point contact, Wear. 271 (2011) 1719-1725. doi:10.1016/j.wear.2010.12.049.

[43] D. He, S. Zheng, J. Pu, G. Zhang, L. Hu, Improving tribological properties of titanium alloys by combining laser surface texturing and diamond-like carbon film, Tribol. Int. 82 (2015) 20-27. doi:10.1016/j.triboint.2014.09.017.

[44] D. Dowson, G.R. Higginson, Elasto-hydrodynamic Lubrication, SI Edition, Pergamon Press Ltd., London, 1977.

[45] X. Lu, M.M. Khonsari, An Experimental Investigation of Dimple Effect on the Stribeck Curve of Journal Bearings, Tribol. Lett. 27 (2007) 169-176. doi:10.1007/s11249-007-9217-x. 
